\title{
Consensus Approach to Secure State Estimation for a Class of Remotely Sensed Networked Systems
}

\author{
Mihalis Tsiakkas*, Nicolas Nicolaou, Marios Polycarpou and Christos Panayiotou
}

\begin{abstract}
Remote sensing is often a crucial part of cyberphysical and networked systems. Measurements taken at remote locations can both serve as monitoring tools as well as to inform control decisions. Over the past few years, a number of cyberattacks were mounted against such systems with severe socio-economic consequences. In this paper we explore how physical measurements can be used in combination with a consensus algorithm, to ensure secure state estimation for a class of tree-like networked systems.
\end{abstract}

\section{INTRODUCTION}

Traditionally, control systems employed hardware components to manipulate the physical processes they were designed to stabilize. With the advent of embedded systems and modern computing, a paradigm shift occurred that saw processes being controlled digitally. This was undoubtedly a vast improvement over the traditional passive mechanical methods. At the same time however, these new technologies exposed those systems to a plethora of cyber threats, that were previously infeasible.

Cyber-Physical Systems (CPSs) integrate physical processes with electronic computing devices and digital communication channels. Often times, CPSs are part of critical infrastructure systems such as power and water distribution networks which are of vital importance to modern society. The severe impact of the unavailability of CPSs, makes such systems an attractive target for malicious entities seeking to inflict economic damage or achieve some political goal.

The number of cyberattack incidents targeting CPSs has been steadily rising over the last few years. Some notable examples include the Maroochy water breach that took place in 2000 [1], the StuxNet worm attack uncovered in 2010 [2], and the Ukrainian power station attack in 2015 [3]. Prevention of such attacks in the future is imperative as they could have severe and devastating consequences.

Many methods have been proposed in the literature to detect and possibly identify cyberattacks on CPSs. For example, a Luenberger observer based approach is suggested by [4], inspired by fault detection and identification techniques. Another method is developed by [5] where the effects of arbitrary data injection attacks of limited cardinality are

\footnotetext{
This work is funded by the European Union's Horizon 2020 research and innovation programme under grant agreement No. 739551 (KIOS CoE) and from the Republic of Cyprus through the Directorate General for European Programmes, Coordination and Development.

The authors are with the KIOS Research and Innovation Center of Excellence and the Department of Electrical and Computer Engineering, University of Cyprus, Nicosia, Cyprus. m.tsiakkas@ieee.org, nicolasnducy.ac.cy, mpolycar@ucy.ac.cy, christosp@ucy.ac.cy.

* Corresponding author.
}

mitigated using error correction techniques and linear programming. A method based on cryptographic keyed-hash functions is developed by [6] offering protection against data substitution attacks. Finally, statistical methods such as the $\chi^{2}$ test [7] have also been employed in the effort of cyberattack detection showing promising results.

State estimation is an integral part of many control systems with uses ranging from plant monitoring to stabilizing feedback control. Therefore, the integrity and correctness of such state estimates is crucial to the normal operation of any control system. This provides a weak point in the security of many systems when state estimation is computed centrally, presenting a single point of failure that can be targeted by an adversary.

The study and development of consensus protocols originated in the field of computer science [8], [9] but quickly found applications in other technical areas, one of which is control theory [10], [11]. Consensus algorithms have been shown in the past to be useful in distributed state estimation [12], security however is rarely considered. The presence of false data is taken into account by [13] though unreasonable limitations are placed on the magnitude of perturbations on the reported state which would render the procedure proposed therein ineffective in many situations.

In this paper a consensus approach is presented for a class of tree-like networked systems which allows for secure state estimation in the presence of cyberattacks on individual nodes. This is achieved by downstream state estimation in combination with upstream estimation error matching. The strength of the proposed method is that each participating vertex relies solely on physical measurements of the relevant signals which cannot be forged or perturbed by a data substitution cyberattack.

The two main contributions of this paper are listed below.

1) Remote observability results are derived for a class of networked systems that exhibit a tree-like structure. Specifically, conditions are given for a parent in the considered graph to be able to estimate the state of its children using only its own input/output data.

2) Based on the above results, a second estimate of the state of each child (given that each child is capable of self state estimation) is obtained. A consensus protocol is developed that exploits this redundancy to detect whether a node has been compromised and suggest which of the two state estimates reported is correct (i.e. that of the parent or that of the child).

The paper is organized as follows. First, the network structure being considered is defined. Then some preliminary 
mathematical results are presented followed by a modeling of the system dynamics. Observability conditions are then derived for this system based on the state space realization previously constructed. Subsequently, a consensus protocol is developed that allows for a subset of nodes in the network to obtain an estimate of the state of a vertex of interest in a secure manner. Finally, a numerical simulation is provided to demonstrate an application of the methodology proposed.

\section{Problem Formulation}

In this paper we consider a special network structure with three layers as shown in Figure 1 where each vertex denotes a CPS. The first layer is termed the generator layer $\mathcal{G}$, which consists of a single linear, time-invariant (LTI) system labeled $G$. The second layer consists of a set $\mathcal{P}$ of so-called consumer nodes, each labeled $P_{i}$, representing a LTI system without any interconnections between them. Each vertex in the first two layers is assumed to be capable of self state estimation. Finally, the third layer contains remote sensors $S_{i, j}$ for the systems in the second layer. Each sensor measures a single element in the output vector of its parent; however, multiple sensors can monitor the same element. For each $P_{i} \in \mathcal{P}$, the corresponding sensor set is defined as $\mathcal{S}_{i}$. The set of all sensors in the third layer is denoted by $\mathcal{S}=\bigcup_{i \in \mathbb{N}_{N}} \mathcal{S}_{i}$.

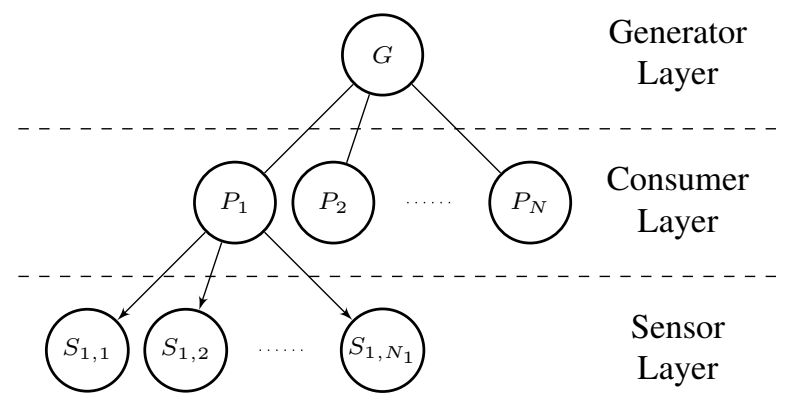

Fig. 1: Example tree graph with 3 levels. Sensors $S_{i, j}$ for $i \in \mathbb{N}_{2, N}$ hidden for clarity.

The edges between the generator and consumer layers of this graph represent a feedback interconnection as shown in Figure 2. It is assumed that the feedback path from the output of $P_{i}$ is not directly measurable at $G$. Conversely, the directed edges between the second and third layer imply that vertices in the sensor layer have no effect on the dynamics of their parent in the consumer layer.

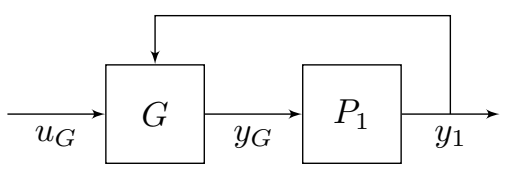

Fig. 2: Edge dynamics.

Feedback structures like the ones depicted above are often encountered in control settings. One example can be found in power distribution networks. Suppose that $G$ is a power station supplying electricity to a number of substations, represented by the nodes in the consumer layer. Note that such systems satisfy the interconnection shown in Figure 2 since an electrical load on a generator induces a mechanical resistance [14]. Finally, vertices in the sensor layer could represent a range of remote devices monitoring the output of each substation (i.e. voltage, frequency, etc.) such as phase measurement units or even domestic electricity meters.

Throughout this paper it is assumed that the communication network overlaying the physical interconnections is complete in the graph theoretic sense [15]. This implies that all vertices can digitally communicate and share information. Additionally, the communication channel between vertices is assumed to be reliable and synchronous, meaning that every message is delivered uncorrupted with a known and constant upper bound on the transmission delay. Furthermore, the physical measurements are also considered to be reliable with the possible inclusion of some noise or disturbances of natural/environmental origin.

Prior to developing a model for the type of attacks being considered herein, we need to define the following notation. $u_{G}$ and $y_{G}$ are the input and output vectors of $G$ respectively, while $x_{i}$ and $y_{i}$ are the state and output vector of $P_{i}$ respectively. The element of $y_{i}$ measured by $S_{i, j}$ is denoted $y_{i, j}$. Additionally, $\tilde{\alpha}(V)$ and $\hat{\alpha}(V)$ are used to denote a measurement and estimate of the variable $\alpha$ respectively, obtained using information available at the vertex $V$. For example, $\hat{x}_{1}(G)$ is the estimate of the state vector of $P_{1}$ generated at $G$.

The adversary is modeled as being able to corrupt or maliciously manipulate a subset of vertices in the network. We denote an adversarial strategy $\mathcal{A}=\mathcal{A}_{B} \cup \mathcal{A}_{C}$, where $\mathcal{A}_{B} \subseteq \mathcal{G} \cup \mathcal{P}$ and $\mathcal{A}_{C} \subset \mathcal{S}$ are the set of vertices that can be attacked by the adversary. Any vertex in $\mathcal{A}_{B}$ is subject to a data substitution attack (i.e. reporting false data) while the ones in $\mathcal{A}_{C}$ are subject to an availability attack (i.e. the adversary blocks them from performing any computation or transmitting data; for example using a DoS attack [16]).

This paper addresses the following problem:

"Given a networked system of the form depicted in Figure 1] obtain a state estimate for each $P_{i} \in \mathcal{P}$ that is sufficiently close to the true value and that is agreed upon by all non-compromised vertices $S_{i, j} \in \mathcal{S}_{i}$ subject to an adversarial strategy $\mathcal{A}$."

This problem can be more formally formulated as follows. For each $P_{i} \in \mathcal{P}$ find $\check{x}_{i}$ such that $\left\|x_{i}-\check{x}_{i}\right\| \leq \epsilon$ for some sufficiently small $0<\epsilon \in \mathbb{R}$ and guarantee that

$$
\hat{x}_{i}\left(S_{i, j}\right)=\check{x}_{i} \quad \forall S_{i, j} \in \mathcal{S}_{i}: S_{i, j} \notin \mathcal{A} .
$$

\section{Preliminaries AND DyNAMic Modeling}

\section{A. Preliminaries}

A few preliminary mathematical facts will now be presented. The set of natural numbers is denoted by $\mathbb{N}$; additionally, $\overline{\mathbb{N}}=\mathbb{N} \cup\{0\}$. Similarly, $\mathbb{R}$ and $\mathbb{C}$ are used to denote the field of real numbers and the complex plane respectively. For any number $n \in \mathbb{N}$ we define $\mathbb{N}_{n}=\{x \in \mathbb{N}: x \leq n\}$ (resp. $\overline{\mathbb{N}}_{n}=\{x \in \overline{\mathbb{N}}: x \leq n\}$ ) as the subset of numbers in $\mathbb{N}($ resp. $\overline{\mathbb{N}})$ upto and including $n$. 
Let $A \in \mathbb{R}^{m \times n}$, then $A^{T}$ and $\operatorname{rank} A$ denote the transpose and rank of $A$ respectively. If $m=n$, then $\operatorname{det} A$ is the determinant of $A$ while its spectrum is denoted by $\Lambda(A)$.

The following notation is adapted from [17]. Given two matrices $A_{1} \in \mathbb{R}^{n_{1} \times m_{1}}$ and $A_{2} \in \mathbb{R}^{n_{2} \times m_{2}}, A_{1} \oplus A_{2} \in$ $\mathbb{R}^{\left(n_{1}+n_{2}\right) \times\left(m_{1}+m_{2}\right)}$ is used to denote the diagonal concatenation of the two given by $A_{1} \oplus A_{2}=\left[\begin{array}{cc}A_{1} & 0 \\ 0 & A_{2}\end{array}\right]$; this notation is extended to matrix sequences with $\bigoplus A_{i}=$ $A_{1} \oplus A_{2} \oplus \cdots \oplus A_{n}$.

The notation $P=\left[\begin{array}{c|c}A & B \\ \hline C & D\end{array}\right]$ is used as a shorthand for the state space representation of the transfer matrix $P=$ $C(s I-A)^{-1} B+D$.

\section{B. System Dynamics Modeling}

We now model the dynamics of the system under consideration; specifically, we are interested in the dynamic mapping from the input to the output of $G$ when taking into account the dynamics of the consumer layer vertices. Note that since the sensor layer does not affect the consumer layer its dynamics (if any) can be ignored.

The generator vertex $G$ is given the minimal state space realization

$$
G=\left[\begin{array}{c|cccc}
A_{G} & B_{G} & B_{G, 1} & \cdots & B_{G, N} \\
\hline C_{G} & D_{G} & D_{G, 1} & \cdots & D_{G, N}
\end{array}\right],
$$

where $A_{G} \in \mathbb{R}^{n_{G} \times n_{G}}$ and $\left[\begin{array}{ll}A_{G} & B_{G} \\ C_{G} & D_{G}\end{array}\right] \in \mathbb{R}^{\left(n_{G}+p_{G}\right) \times\left(n_{G}+q_{G}\right)}$. In this realization $B_{G, i} \in \mathbb{R}^{n_{G} \times p_{i}}$ and $D_{G, i} \in \mathbb{R}^{p_{G} \times p_{i}}$ describe how the output of $P_{i}$ affects the states and output of $G$ respectively.

Similarly to $G$, every vertex $P_{i} \in \mathcal{P}$ is assumed to have the minimal state space realization

$$
P_{i}=\left[\begin{array}{c|c}
A_{i} & B_{i} \\
\hline C_{i} & D_{i}
\end{array}\right]
$$

where $A_{i} \in \mathbb{R}^{n_{i} \times n_{i}}$ and $\left[\begin{array}{ll}A_{i} & B_{i} \\ C_{i} & D_{i}\end{array}\right] \in \mathbb{R}^{\left(n_{i}+p_{i}\right) \times\left(n_{i}+p_{G}\right)}$.

We now move on to modeling the network dynamics as a whole. First define the following

$$
\begin{gathered}
B_{\bullet}=\left[\begin{array}{c}
B_{1} \\
\vdots \\
B_{N}
\end{array}\right], D_{\bullet}=\left[\begin{array}{c}
D_{1} \\
\vdots \\
D_{N}
\end{array}\right], \\
B_{G, \bullet}=\left[\begin{array}{llll}
B_{G, 1} & \cdots & B_{G, N}
\end{array}\right], D_{G, \bullet}=\left[\begin{array}{lll}
D_{G, 1} & \cdots & D_{G, N}
\end{array}\right], \\
C_{\bullet}=\bigoplus_{i \in \mathbb{N}_{N}} C_{i} \text { and } R=I-D_{G, \bullet} D_{\bullet}
\end{gathered}
$$

Finally, define

$$
\begin{gathered}
\tilde{A}=\left[\begin{array}{cc}
A_{G} & B_{G, \bullet} C \bullet \\
0 & \underset{i \in \mathbb{N}_{N}}{\bigoplus} A_{i}
\end{array}\right], \\
\tilde{B}=\left[\begin{array}{c}
B_{G}+B_{G, \bullet} D_{\bullet} R^{-1} D_{G} \\
B \bullet R^{-1} D_{G}
\end{array}\right], \\
\text { and } \tilde{C}=R^{-1}\left[\begin{array}{ll}
C_{G} & D_{G, \bullet} C
\end{array}\right] .
\end{gathered}
$$

Then the over all dynamics of the network from the input of $G$ to its output can be expressed as

$$
\Sigma=\left[\begin{array}{c|c}
\tilde{A}+\left[\begin{array}{c}
B_{G, \bullet} D \bullet \\
B \bullet
\end{array}\right] \tilde{C} & \tilde{B} \\
\hline \tilde{C} & D_{G}
\end{array}\right] .
$$

Note that the above model requires that $\operatorname{det} R \neq 0$. Though this might seem like a significant restriction it in fact amounts to the well-posedness of the feedback interconnection [18]. Well-posedness is a crucial aspect of modeling any physically realizable system and ensures the properness of the resulting transfer matrix.

\section{Generator to Consumer State Observability}

As previously stated, our approach relies on downstream state estimation to obtain an estimate of the states of each child of $G$. The observability of the states of the consumer layer nodes $P_{i}$ from the generator vertex $G$ is therefore a crucial property for the applicability of the technique developed herein.

Tools developed in the context of structural controllability (and by duality observability) [19], [20] can be used to show that (6) is observable for some choice of parameters. This however does not help in establishing the observability of a given system. No studies of the system structure being considered herein can be found in the literature. This necessitates the development of custom observability tests.

Note that the state space realization of $\Sigma$ given by (6) is observable if and only if $(\tilde{C}, \tilde{A})$ is observable. This is a consequence of the fact that observability is invariant under state estimation [18] upon employing $-\left[\begin{array}{c}B_{G, \bullet} D_{\bullet} \\ B_{\bullet}\end{array}\right]$ as a (not necessarily stabilizing) gain matrix. Now suppose that $\tilde{L}$ is a stabilizing state estimation gain matrix for $(\tilde{C}, \tilde{A})$ (i.e. $\tilde{A}+$ $\tilde{L} \tilde{C}$ is Hurwitz) and define $L=\tilde{L}-\left[\begin{array}{c}B_{G, \bullet} D \\ B \bullet\end{array}\right]$. Then it is simple to show that $L$ is a stabilizing state estimation gain for the original state space realization of $\Sigma$ given by (6). The combination of the above two facts now implies that there is no loss of generality nor any significant increase in complexity in studying the observability of $(\tilde{C}, \tilde{A})$ over that of the original system.

The main result of this section concerns the observability of $\Sigma$ as given in (6). A necessary and sufficient condition is presented in the following theorem.

Theorem 1: Consider $\Sigma$ as given by (6) and suppose that the following assumptions hold:

A1. $\Lambda\left(A_{G}\right) \cap \Lambda\left(A_{i}\right)=\emptyset \quad \forall i \in \mathbb{N}_{N}$,

A2. $\Lambda\left(A_{i}\right) \cap \Lambda\left(A_{j}\right)=\emptyset \quad \forall\{i, j\} \subseteq \mathbb{N}_{N}$, and

A3. $\left(\left[\begin{array}{l}B_{G, i} \\ D_{G, i}\end{array}\right] C_{i}, A_{i}\right)$ is observable $\forall i \in \mathbb{N}_{N}$.

Furthermore, let $X_{i} \in \mathbb{R}^{n_{G} \times n_{i}}$ be the solution to the Sylvester equation

$$
A_{G} X_{i}-X_{i} A_{i}+B_{G, i} C_{i}=0 .
$$


Then (6) is observable if and only if

$$
\left(C_{G} X_{i}+D_{G, i} C_{i}, A_{i}\right) \text { is observable } \forall i \in \mathbb{N}_{N} .
$$

Proof: We prove the result by considering the observability of the pair $(\tilde{C}, \tilde{A})$. Due to assumption $\mathbf{A 1}$ and via [17, Theorem 2.4.4.1], a solution to (7) exists for all $i \in \mathbb{N}_{N}$. Now define the matrices $X=\left[\begin{array}{lll}X_{1} & \cdots & X_{n}\end{array}\right]$ and $T=\left[\begin{array}{cc}I & -X \\ 0 & I\end{array}\right]$. Since $\operatorname{det} T \neq 0$ and $\operatorname{det} R \neq 0$ it follows that for all $s \in \mathbb{C}$

$$
\operatorname{rank}\left[\begin{array}{c}
\tilde{A}-s I \\
\tilde{C}
\end{array}\right]=\operatorname{rank}\left(\left[\begin{array}{cc}
T & 0 \\
0 & R
\end{array}\right]\left[\begin{array}{c}
\tilde{A}-s I \\
\tilde{C}
\end{array}\right] T^{-1}\right) .
$$

Furthermore, note that

$$
\begin{aligned}
& {\left[\begin{array}{c}
T(\tilde{A}-s I) T^{-1} \\
R \tilde{C} T^{-1}
\end{array}\right]=\left[\begin{array}{ccc}
A_{G}-s I & 0 \\
0 & \multicolumn{2}{c}{\bigoplus_{i \in \mathbb{N}_{N}} A_{i}-s I} \\
C_{G} & \multicolumn{2}{c}{C_{G} X+D_{G, \bullet} C_{\bullet}}
\end{array}\right]} \\
& =\left[\begin{array}{cccc}
A_{G}-s I & 0 & \cdots & 0 \\
0 & A_{1}-s I & \cdots & 0 \\
\vdots & \vdots & \ddots & \vdots \\
0 & 0 & \cdots & A_{N}-s I \\
C_{G} & C_{G} X_{1}+D_{G, 1} C_{1} & \cdots & C_{G} X_{N}+D_{G, N} C_{N}
\end{array}\right]
\end{aligned}
$$

Finally, due to assumption $\mathbf{A 2}$ and via the standard PBH observability tests, it follows that $(\tilde{C}, \tilde{A})$ is observable if and only if $\left(C_{G}, A_{G}\right)$ and $\left(C_{G} X_{i}+D_{G, i} C_{i}, A_{i}\right)$ are observable for all $i \in \mathbb{N}_{N}$ which concludes the proof.

The observability assumption $\mathbf{A 3}$ imposed in the above theorem, though not invoked in the proof, can be shown to be necessary for the observability of the overall system. This fact is proven in the following lemma.

Lemma 2: The state space realization for $\Sigma$ given in (6) is observable only if $\left(\left[\begin{array}{c}B_{G, i} \\ D_{G, i}\end{array}\right] C_{i}, A_{i}\right)$ is observable $\forall i \in \mathbb{N}_{N}$.

Proof: Using the standard PBH test for observability we have that (6) is observable if and only if

$$
\begin{aligned}
\operatorname{rank} & {\left[\begin{array}{c}
\tilde{A}-s I \\
\tilde{C}
\end{array}\right]=n_{G}+\sum_{i \in \mathbb{N}_{N}} n_{i} \quad \forall s \in \mathbb{C} } \\
& \Rightarrow\left(\begin{array}{c}
\operatorname{rank}\left[\begin{array}{c}
A_{i}-s I \\
B_{G, i} C_{i} \\
R^{-1} D_{G, i} C_{i}
\end{array}\right]=n_{i} \forall i \in \mathbb{N}_{N} \\
\text { and } \operatorname{rank}\left[\begin{array}{c}
A_{G}-s I \\
R^{-1} C_{G}
\end{array}\right]=n_{G}
\end{array}\right) \forall s \in \mathbb{C} .
\end{aligned}
$$

It can now be observed that the above is precisely one of the standard PBH tests for observability for the pairs $\left(R^{-1} C_{G}, A_{G}\right)$ and $\left(\left[\begin{array}{c}B_{G, i} \\ R^{-1} D_{G, i}\end{array}\right] C_{i}, A_{i}\right)$. The proof then follows by noting that since $R$ is non-singular, the term $R^{-1}$ does not affect the observability of the pair.

It can also be shown that a sufficient condition for $\left(\left[\begin{array}{c}B_{G, i} \\ D_{G, i}\end{array}\right] C_{i}, A_{i}\right)$ to be observable is that rank $\left[\begin{array}{l}B_{G, i} \\ D_{G, i}\end{array}\right]=p_{i}$. Hence, if $p_{i}=1$ (i.e. $P_{i}$ has a single output), then assumption A3 of Theorem 1 is satisfied if and only if $\left[\begin{array}{c}B_{G, i} \\ D_{G, i}\end{array}\right] \neq 0$.
Once the observability of the system under consideration is established using Theorem 1, the design of an observer can be tackled using standard techniques found in the literature.

Remark 1: Assumptions A1 and A2 of Theorem 1 could be relaxed. In that case however, the geometric multiplicities of the shared eigenvalues must be taken into account when testing for observability [21, Lemma 10].

\section{Secure State Estimation via Consensus}

In this section we examine how we can implement a consensus protocol, in order to guarantee that the state of the vertex of interest is securely and correctly estimated. For simplicity, we only consider the subgraph of Figure 1 consisting of $G, P_{1}$ and all of $S_{1, i}$. Furthermore, it is assumed that the observability conditions outlined in Theorem 1 hold true and therefore the states of $P_{1}$ can be estimated at $G$ without any exogenous information. A high level description of the proposed procedure is given in Algorithm 1 below.

Algorithm 1 (Overview):

a) Using $\tilde{u}_{G}(G)$ and $\tilde{y}_{G}(G), G$ generates and sends the pair $\left\{\tilde{y}_{G}(G), \hat{x}_{1}(G)\right\}$ to all $S_{1, i} \in \mathcal{S}_{1}$.

b) Using $\tilde{y}_{G}\left(P_{1}\right)$ and $\tilde{y}_{1}\left(P_{1}\right), P_{1}$ generates and sends the pair $\left\{\tilde{y}_{G}\left(P_{1}\right), \hat{x}_{1}\left(P_{1}\right)\right\}$ to all $S_{1, i} \in \mathcal{S}_{1}$.

c) Each of $S_{1, i}$ calculates $0 \leq \phi\left(S_{1, i} \mid G\right) \in \mathbb{R}$ and $0 \leq$ $\phi\left(S_{1, i} \mid P_{1}\right) \in \mathbb{R}$, where for $X \in\left\{G, P_{1}\right\}$,

$$
\phi\left(S_{1, i} \mid X\right)=\left|\tilde{y}_{1, i}\left(S_{1, i}\right)-\Gamma_{1, i}^{T}\left[\begin{array}{ll}
C_{1} & D_{1}
\end{array}\right]\left[\begin{array}{c}
\hat{x}_{1}(X) \\
\tilde{y}_{G}(X)
\end{array}\right]\right|,
$$

and $\Gamma_{1, i} \in \mathbb{N}^{p_{1}}$ is a binary vector indicating which output of $P_{1}$ is measured by $S_{1, i}$ (i.e. $y_{1, i}=\Gamma_{1, i}^{T} y_{1}$ ).

d) The consensus input of $S_{1, i}$ denoted $\psi\left(S_{1, i}\right) \in \mathbb{N}_{2}$ is set according to

$$
\psi\left(S_{1, i}\right)=v\left(\phi\left(S_{1, i} \mid P_{1}\right)-\epsilon\right)\left[1+v\left(\phi\left(S_{1, i} \mid G\right)-\epsilon\right)\right],
$$

where $0<\epsilon \in \mathbb{R}$ is a small positive constant used as a threshold and $v: \mathbb{R} \mapsto \overline{\mathbb{N}}_{1}$ denotes the unit step function 1

e) A consensus protocol $\mathcal{C}: \overline{\mathbb{N}}_{2}^{N_{1}} \mapsto \overline{\mathbb{N}}_{2}$ is executed among $\mathcal{S}_{1}$ with inputs $\psi\left(S_{1, i}\right)$ and output $\psi\left(\mathcal{S}_{1}\right) \in \overline{\mathbb{N}}_{2}$.

f) Finally, $\hat{x}_{1}\left(\mathcal{S}_{1}\right)$ (i.e. the globally agreed upon state estimate of $\left.P_{1}\right)$ is chosen according to

$$
\hat{x}_{1}\left(\mathcal{S}_{1}\right)=\left\{\begin{array}{cl}
\hat{x}_{1}\left(P_{1}\right) & \text { if } \psi\left(\mathcal{S}_{1}\right)=0, \\
\hat{x}_{1}(G) & \text { if } \psi\left(\mathcal{S}_{1}\right)=1, \\
\infty & \text { if } \psi\left(\mathcal{S}_{1}\right)=2 .
\end{array}\right.
$$

In the third case where $\psi\left(\mathcal{S}_{1}\right)=2$, the value of $\hat{x}_{1}\left(\mathcal{S}_{1}\right)$ is set to $\infty$, denoting that none of the received state estimates can be trusted and thus alternative contingencies must be executed.

A candidate for $\mathcal{C}$ is presented in Algorithm 2.

Algorithm 2 (Consensus Protocol $\mathcal{C}$ ):

Let $r \in \mathbb{N}$ denote the current round starting with $r=1$. For notational brevity we define the set $\mathcal{S}_{1}^{r} \subseteq \mathcal{S}_{1}$ as the set of sensors $S_{1, i}$ that are active (i.e. have not been compromised) at round $r$; note that at the start of the algorithm $\mathcal{S}_{1}^{1}=\mathcal{S}_{1}$.

\footnotetext{
${ }^{1}$ The unit step function is defined as $v(x)= \begin{cases}0 & \text { if } x \leq 0, \\ 1 & \text { otherwise. }\end{cases}$
} 
At each $S_{1, i}$, the following algorithm is executed:

a) Send $\psi\left(S_{1, i}\right)$ to all $S_{1, j} \in \mathcal{S}_{1}^{r}$.

b) Let $\mathcal{L}^{r}=\left\{l_{k} \in \mathbb{N}_{N_{1}}: S_{1, l_{k}} \in \mathcal{S}_{1}^{r}\right\} \subseteq \mathbb{N}_{N_{1}}$ and construct the row vector

$$
\Psi_{1, i}^{r}=\left[\begin{array}{lll}
\psi\left(S_{1, l_{1}}\right) & \cdots & \psi\left(S_{1, l_{\left|\mathcal{S}_{1}^{r}\right|}}\right)
\end{array}\right] \quad \forall l_{k} \in \mathcal{L}^{r} .
$$

c) Set $\psi\left(S_{1, i}\right)=\left\|\Psi_{1, i}^{r}\right\|_{\infty}$.

d) If $\left|\mathcal{S}_{1}^{r}\right|=\left|\mathcal{S}_{1}^{r-1}\right|, \sqrt{\left|\mathcal{S}_{1}^{r}\right|} \psi\left(S_{1, i}\right)=\left\|\Psi_{1, i}^{r}\right\|$ and $\Psi_{1, i}^{r}=$ $\Psi_{1, i}^{r-1}$ decide $\psi\left(S_{1, i}\right)$; otherwise increment $r$ and go to step $a$.

The effect of the above algorithm is that all sensors agree on the maximum value proposed by any sensor in $\mathcal{S}_{1}$ at the start (i.e. $\psi\left(\mathcal{S}_{1}\right)=\max _{S_{1, i} \in \mathcal{S}_{1}} \psi\left(S_{1, i}\right)$ ). Hence, if an abnormality is detected by any sensor then this will be reflected in the output of the consensus protocol.

\section{Analysis \& Discussion}

The proposed approach (as presented) is not without flaws. We begin our discussion with some possible drawbacks. An obvious and serious one is the fact that any additive perturbations to the true input and state vector estimate lying in $\operatorname{ker}\left(\Gamma_{1, i}^{T}\left[\begin{array}{ll}C_{1} & D_{1}\end{array}\right]\right)$ would not affect $\phi\left(S_{1, i} \mid G\right)$ or $\phi\left(S_{1, i} \mid P_{1}\right)$. To see this, suppose that $\hat{x}_{1}\left(P_{1}\right)$ is a true estimation of $x_{1}\left(P_{1}\right)$ and let $v \in \operatorname{ker} C_{1}$; then $C_{1}\left(\hat{x}_{1}\left(P_{1}\right)+v\right)=$ $C_{1} \hat{x}_{1}\left(P_{1}\right)$. Hence a malicious adversary could substitute the true state estimate without affecting the input to the consensus protocol. This problem will be addressed in a future publication.

Another issue is that the use of the threshold $\epsilon$ would allow an attacker to remain undetected by only perturbing the true values by small amounts. This however is not unique to our solution as many methods found in the literature also suffer from this problem. Such an attack is termed as $(\epsilon, \mathbf{s})$ effective by [22] and is guaranteed to exists in any observer based attack detection method.

\section{NUMERICAL EXAMPLE}

We now present a simple numerical example to demonstrate the use of the techniques developed in the previous sections. The network being considered in this example has $N=2$ and $N_{1}=N_{2}=4$. Hence the consumer layer consists of two vertices, each of which has four remote sensors. The dimensions of the vertices in the generator and consumer layer are given by $p_{G}=q_{G}=1$ and $p_{1}=p_{2}=2$. Two sensors are assigned to monitor each output of $P_{1}$ and $P_{2}$. Specifically, $S_{i, 1}$ and $S_{i, 2}$ measure $y_{i, 1}$ while $S_{i, 3}$ and $S_{i, 4}$ measure $y_{i, 2}$.

The dynamics of each vertex in the first two layers were randomly generated and given by

$$
G=\left[\begin{array}{ccc|c}
-0.967 & 0.500 & -0.604 & 0.563 \\
-0.548 & -0.985 & -0.819 & -0.853 \\
0.561 & 0.849 & -0.988 & 0 \\
\hline 0 & -0.825 & 0 & 0
\end{array}\right]
$$

$$
P_{1}=\left[\begin{array}{ccc|c}
-0.967 & 0.500 & -0.604 & 0.604 \\
-0.548 & -0.985 & -0.819 & 1.586 \\
0.561 & 0.849 & -0.988 & -0.858 \\
\hline-0.286 & -0.107 & 0.758 & 0 \\
-2.264 & 0.831 & 0.758 & 0
\end{array}\right],
$$

and

$$
P_{2}=\left[\begin{array}{ccc|c}
-2.670 & 1.523 & 2.574 & -0.562 \\
-2.847 & -1.218 & -1.461 & 0 \\
0.917 & 2.814 & -2.746 & -0.221 \\
\hline 1.158 & 0.578 & 0 & 0 \\
-0.696 & -0.110 & 0.664 & 0
\end{array}\right] .
$$

Note that all three systems are spectrally disjoint thus satisfying assumptions item A1 and item A2 of Theorem 1. The interconnection matrices were given by $D_{G, 1}=0, D_{G, 2}=0$,

$$
B_{G, 1}=-\left[\begin{array}{ll}
3.497 & 3.306 \\
5.246 & 1.102 \\
1.749 & 2.204
\end{array}\right] \text { and } B_{G, 2}=\left[\begin{array}{ll}
1.420 & 0.501 \\
4.260 & 1.002 \\
2.840 & 1.503
\end{array}\right]
$$

Note that both have full column rank, therefore satisfying assumption A3 of Theorem 1 .

With the above data, the solutions of the Sylverster equation defined in (7) were obtained as

$$
X_{1}=\left[\begin{array}{ccc}
-3.18 & 1.03 & -0.84 \\
-3.16 & -0.33 & -0.63 \\
0.18 & 1.66 & -0.66
\end{array}\right] \text { and } X_{2}=\left[\begin{array}{ccc}
0.15 & -0.52 & 0.14 \\
0.24 & -1.24 & 0.99 \\
0.12 & -0.99 & -0.10
\end{array}\right] \text {. }
$$

It can now be confirmed via Theorem 1 that both vertices in the consumer layer are indeed observable using only the input and output data of $G$.

The state estimation gain was then set to

$$
\begin{gathered}
L=\left[\begin{array}{lll}
L_{G}^{*} & L_{1}^{*} & L_{2}^{*}
\end{array}\right]^{*}, \text { where } L_{G}=\left[\begin{array}{c}
16740.558 \\
12472.741 \\
13120.623
\end{array}\right], \\
L_{1}=-\left[\begin{array}{c}
3.256 \\
4.612 \\
20006.594
\end{array}\right] \text { and } L_{2}=\left[\begin{array}{c}
-7.654 \\
28.985 \\
3.393
\end{array}\right] .
\end{gathered}
$$

To avoid numerical issues, a centralized stabilizing feedback was used utilizing the state estimations of the designed observer. Due to the separation principle, this does not affect the rest of the results presented. The stabilizing gain used was

$$
\begin{gathered}
F=\left[\begin{array}{lll}
F_{G} & F_{1} & F_{2}
\end{array}\right] \text { where } F_{G}=\left[\begin{array}{lll}
-0.29 & 23.21 & -4.08
\end{array}\right], \\
F_{1}=\left[\begin{array}{lll}
6.56 & 2.58 & 272.87
\end{array}\right], \text { and } F_{2}=\left[\begin{array}{lll}
5.33 & -0.88 & -0.36
\end{array}\right] .
\end{gathered}
$$

With these parameters, a simulation was performed producing the results shown in Figures 3 to 5 . This simulation lasts for $20 \mathrm{~s}$ and contains two attacks. The first attack is against $P_{1}$ and lasts for $10 \mathrm{~s}$ starting at $t=5 \mathrm{~s}$. For the first $7 \mathrm{~s}$ of the attack, the last element of $\hat{x}_{1}\left(P_{1}\right)$ is additively perturbed while for the last $7 \mathrm{~s}$ the first element of $\hat{x}_{1}(G)$ is also corrupted. Another attack is mounted against $P_{2}$ from $t=8 \mathrm{~s}$ to $t=15 \mathrm{~s}$ during which time all of $\hat{x}_{2}\left(P_{2}\right)$ is distorted. Additionally, $S_{i, 1}$ and $S_{i, 3}$ crash (i.e. fail to transmit $\psi\left(S_{i, j}\right)$ ) over the intervals $t \in[10,13] s$ for $S_{1, j}$ and $t \in[11,16] s$ for $S_{2, j}$.

In all plots, the blue and red lines are associated with the subgraph containing $P_{1}$ and $P_{2}$ respectively. It can be seen 


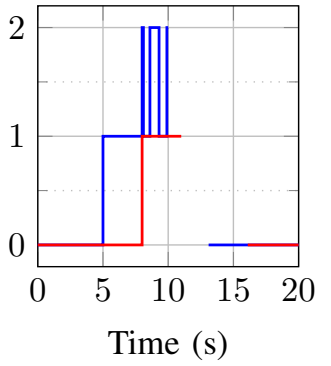

(a) $\psi\left(S_{i, 1}\right)$

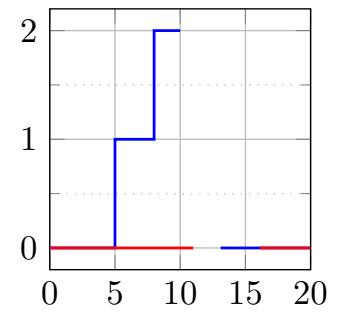

Time (s)

(c) $\psi\left(S_{i, 3}\right)$

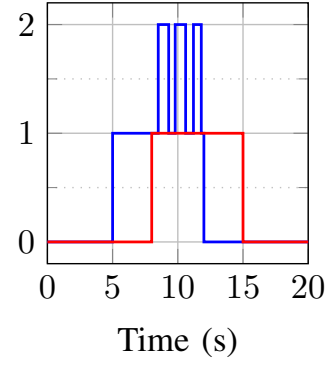

(b) $\psi\left(S_{i, 2}\right)$

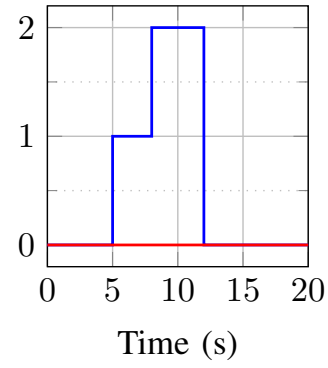

(d) $\psi\left(S_{i, 4}\right)$
Fig. 3: Consensus input $\psi\left(S_{i, j}\right)$.

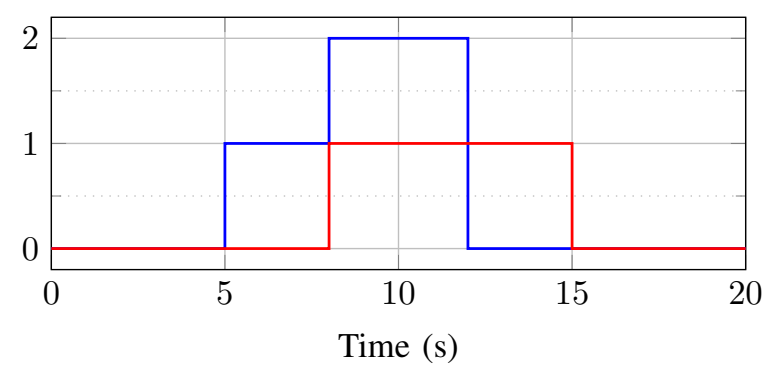

Fig. 4: Consensus output $\psi\left(\mathcal{S}_{i}\right)$.

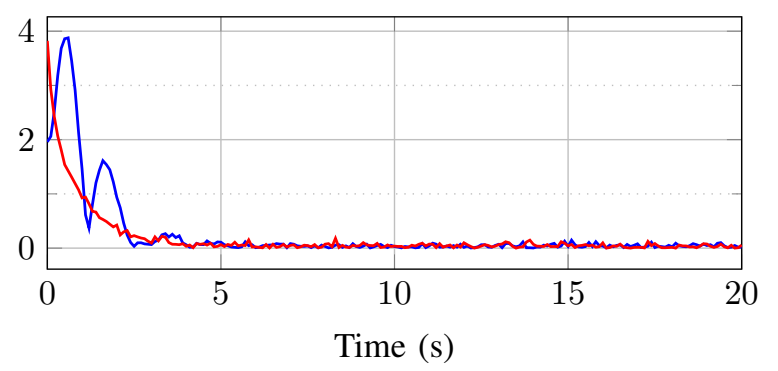

Fig. 5: State estimation error $\left\|\hat{x}_{i}(G)-x_{i}\right\|$.

from these figures that the proposed methodology correctly identifies the existence of the attacks. In the case of $\mathcal{S}_{1}$, as soon as $\hat{x}_{1}\left(P_{1}\right)$ is corrupted by the adversary, the algorithm suggests using $\hat{x}_{1}(G)$ with $\psi\left(\mathcal{S}_{1}\right)=1$. When $\hat{x}_{1}(G)$ is also found to be faulty at $t=8 \mathrm{~s}$ we see that $\psi\left(\mathcal{S}_{1}\right)=2$ (i.e. none of the received measurements can be trusted). When the attack on $\hat{x}_{1}\left(P_{1}\right)$ ends at $t=12 \mathrm{~s}$, it can be observed that the output of the consensus returns to $\psi\left(\mathcal{S}_{1}\right)=0$ even though the attack on $\hat{x}_{1}(G)$ persists. Furthermore, when the attack against $\hat{x}_{2}\left(P_{2}\right)$ is active, the consensus protocol returns $\psi\left(\mathcal{S}_{2}\right)=1$ as expected; even though $S_{2,3}$ and $S_{2,4}$ are not affected by the attack.

\section{CONCLUSIONS}

In this paper a protocol to establish secure state estimation for a class of networked cyber-physical systems has been developed utilizing tools from both control theory and computer science. Network observability conditions for the class of systems being considered were presented. A consensus algorithm was then constructed ensuring that any abnormalities in the received data are detected. Finally, the proposed techniques were validated using numerical simulations.

\section{REFERENCES}

[1] J. Slay and M. Miller. Lessons learned from the maroochy water breach. Critical infrastructure protection, pages 73-82, 2007.

[2] J. P. Farwell and R. Rohozinski. Stuxnet and the future of cyber war. Survival, 53:23-40, 022011.

[3] G. Liang, S. R. Weller, J. Zhao, F. Luo, and Z. Y. Dong. The 2015 ukraine blackout: Implications for false data injection attacks. IEEE Transactions on Power Systems, 32(4):3317-3318, July 2017.

[4] F. Pasqualetti, F. Dörfler, and F. Bullo. Attack detection and identification in cyber-physical systems. IEEE Transactions on Automatic Control, 58(11):2715-2729, Nov 2013.

[5] H. Fawzi, P. Tabuada, and S. Diggavi. Secure estimation and control for cyber-physical systems under adversarial attacks. IEEE Transactions on Automatic Control, 59(6):1454-1467, June 2014.

[6] M. Tsiakkas, P. Kolios, M. Polycarpou, and C. Panayiotou. Establishing data integrity in networks of cyber-physical systems. In 2018 European Control Conference (ECC), pages 350-355, Limassol, Cyprus, June 2018.

[7] I. Jovanov and M. Pajic. Sporadic data integrity for secure state estimation. In 2017 IEEE 56th Annual Conference on Decision and Control (CDC), pages 163-169, Dec 2017.

[8] M. Pease, R. Shostak, and L. Lamport. Reaching agreement in the presence of faults. J. ACM, 27(2):228-234, April 1980.

[9] L. Lamport, R. Shostak, and M. Pease. The byzantine generals problem. ACM Trans. Program. Lang. Syst., 4(3):382-401, July 1982.

[10] S. Sundaram and C. N. Hadjicostis. Distributed function calculation via linear iterative strategies in the presence of malicious agents. IEEE Transactions on Automatic Control, 56(7):1495-1508, July 2011.

[11] J. Wang, A. Lanzon, and I. R. Petersen. Robust output feedback consensus for networked negative-imaginary systems. IEEE Transactions on Automatic Control, 60(9):2547-2552, September 2015.

[12] S. Kar, G. Hug, J. Mohammadi, and J. M. F. Moura. Distributed state estimation and energy management in smart grids: A consensus+ innovations approach. IEEE Journal of Selected Topics in Signal Processing, 8(6):1022-1038, Dec 2014.

[13] R. H. Etemad and F. Lahouti. Resilient decentralized consensus-based state estimation for smart grid in presence of false data. In 2016 IEEE International Conference on Acoustics, Speech and Signal Processing (ICASSP), pages 3466-3470, March 2016.

[14] Y. Hase. Handbook of Power System Engineering. John Wiley \& Sons, Ltd, apr 2007.

[15] G. Chartrand and P. Zhang. A First Course in Graph Theory. Dover books on mathematics. Dover Publications, 2012.

[16] Claudio De Persis and Pietro Tesi. Input-to-state stabilizing control under denial-of-service. IEEE Transactions on Automatic Control, 60(11):2930-2944, Nov 2015.

[17] R. A. Horn and C.R. Johnson. Matrix Analysis. Cambridge University Press, 2nd edition, 2012.

[18] K. Zhou, J. C. Doyle, and K. Glover. Robust and Optimal Control. Prentice-Hall, Inc., Upper Saddle River, New Jersey, 1996.

[19] Ching-Tai L. Structural controllability. IEEE Transactions on Automatic Control, 19(3):201-208, June 1974.

[20] J. L. Willems. Structural controllability and observability. Systems \& Control Letters, 8(1):5 - 12, 1986.

[21] M. Tsiakkas and A. Lanzon. Foundations of a bicoprime factorization theory. IEEE Transactions on Automatic Control, 62(9):4598-4603, Sept 2017.

[22] S. Mishra, Y. Shoukry, N. Karamchandani, S. N. Diggavi, and P. Tabuada. Secure state estimation against sensor attacks in the presence of noise. IEEE Transactions on Control of Network Systems, 4(1):49-59, March 2017. 